\title{
Systemic treatment of liver metastases from colorectal cancer
}

\author{
Alexander Stein and Hans-Joachim Schmoll
}

\begin{abstract}
Patients presenting with synchronous or metachronous colorectal cancer liver metastases (CLM) should be evaluated for multimodal management with curative intent. Preoperative systemic chemotherapy shows beneficial impact on adjuvant progressionfree survival and also borderline on overall survival, without significantly increasing initially $\mathrm{R} 0$ resectable patients postoperative complication rates. Postoperative chemotherapy recommended based on the perioperative trial experience for those patients achieving at least stable disease during preoperative chemotherapy, or based on the adjuvant trials for patients receiving upfront resection. 'Borderline' resectable CLM, preoperative chemotherapy plays an important role in both in achievement of a resectable status and improvement of prognosis. Recent 4 drug combinations demonstrated response rates up to $80 \%$ even for advanced disease and are thus promising regimens for further evaluation in patients with resectable or unresectable liver-limited (+/- lung) disease.
\end{abstract}

Keywords: colorectal liver metastases, induction treatment, perioperative, resectability

\section{Background}

Colorectal cancer (CRC) is the most frequently diagnosed cancer in Europe and one of the leading causes of cancer death worldwide [Ferlay et al. 2010; Jemal et al. 2011]. One in four patients with CRC presents with metastatic disease at the time of diagnosis and a further quarter of patients will develop metastases after curative resection, of whom $20-30 \%$ present with liver metastases only [Mantke et al. 2012; Van der Pool et al. 2012]. Liver or lung metastases without further sites of disease represent a unique and favourable biology of advanced CRC as metastases can be restricted to these visceral organs for a long time or even forever, making the resection or elimination of these metastases in addition to chemotherapy an important and meaningful tool to enable cure or at least long-term survival.

Upfront stratification of patients with metastatic CRC (mCRC) into clinical groups according to clinical presentation and treatment aim by a multidisciplinary team is important for tailoring the treatment approach especially for either initially or potentially resectable liver \pm lung metastases [Schmoll and Sargent, 2007; Van Cutsem et al. 2010].
Recent retrospective series have demonstrated profound improvement in the outcome of patients with CRC over the past 20 years due to advances in chemotherapy and a dramatic increase in patients undergoing liver resection for either primarily or secondarily resectable colorectal liver metastases (CLM), resulting in an overall survival (OS) comparable with that of stage III [Union for International Cancer Control (UICC)] colon cancer [Kopetz et al. 2009; Morris et al. 2010]. However, with the majority of patients relapsing after liver resection, either intrahepatic (about $70 \%$ ), extrahepatic (about $50 \%$ ) or both, further research in terms of patient selection as well as improved treatment modalities and intensity is urgently warranted [de Jong et al. 2009].

\section{Stratification of patients with CLM}

Patients with hepatic metastases should be stratified in upfront 'clearly R0' resectable CLM and not R0, but potentially resectable CLM after downsizing with (neoadjuvant) chemotherapy. Although the status of the primary tumour in cases of synchronous disease needs to be taken into account, especially in cases of rectal cancer,
Ther Adv Med Oncol (2013) 5(3) 193-203 DOI: $10.1177 /$ 1758834012473347

(c) The Author(s), 2012. Reprints and permissions: http://www.sagepub.co.uk/ journalsPermissions.nav

Correspondence to: Dr Hans Joachim Schmoll, MD, PhD

Department for Oncology/ Haematology, Martin Luther University HalleWittenberg, Ernst Grube Str. 40, 06120 Halle, Germany hans-joachim.schmolla uk-halle.de

Alexander Stein, MD Department of Oncology, Hematology, BMT with Section Pneumology, Hubertus Wald Tumour Centre, University Cancer Centre Hamburg, University Medical Centre Hamburg-Eppendorf, Hamburg, Germany 
a patient's prognosis depends on the extent and resectability of CLM. The treatment approach differs in regard of resectability. Initially resectable patients receive perioperative systemic treatment with fluoropyrimidine and oxaliplatin for up to 6 months to improve prognosis, similar to adjuvant treatment of stage III colon cancer. For not $\mathrm{R} 0$ resectable patients the most active induction chemotherapy, for as long as necessary and as short as possible, is required to ensure achievement of secondary $\mathrm{R} 0$ resectability.

\section{Prognostic factors for liver resection of CLM}

Already in the late 1980s, risk factors for poor outcome after resection of clearly R0 resectable CLM were identified, e.g. presence of positive portal lymph nodes, extrahepatic metastases, four or more hepatic metastases or primary tumour stage Duke's C [Hughes et al. 1986]. The scoring system described by Fong and colleagues includes five risk factors: node-positive primary, diseasefree interval $<12$ months, $>1$ lesion, size $>5 \mathrm{~cm}$, and carcinoembryonic antigen (CEA) $>200 \mathrm{ng} / \mathrm{m}$ [Fong et al. 1999]. Patients with five risk factors had a 5 -year OS rate (5-yOSR) of $14 \%$ with a median of 22 months compared with a 5-yOSR of $44 \%$ and a median of 51 months in the presence of one risk factor. The Fong score, however, is only validated for upfront resectable CLM and does not include preoperative chemotherapy, which limits the use of this score for the current treatment situation.

Recently, the international liver metastases survey, including data for about 4500 patients with preoperatively treated CLM, confirmed the poor prognosis of patients with lymph node positive primary, abnormal CEA levels and number of CLM, although the cutoff value was three in the current analyses. Furthermore, more than one line and six cycles of preoperative treatment, incomplete macroscopic resection (R2), concomitant extrahepatic metastases and progression during preoperative chemotherapy were associated with poor prognoses [Adam and Barroso, 2011].

Besides clinicopathological variables, molecular markers might gain importance for prognostic information on patients selected for surgery. The worse prognosis of BRAF-mutated mCRC has been shown in several trials with a progressionfree survival (PFS) of about 3-6 months and an OS of up to 15 months, although patient numbers are limited by the low frequency [Bokemeyer et al. 2012; Price et al. 2011; Tol et al. 2009a]. Recent analysis indicated that upfront intensive four-drug treatment, FOLFOXIRI (irinotecan, oxaliplatin, fluorouracil and folinate) and bevacizumab, for BRAF-mutant mCRC might result in more favourable PFS (11.8 months) and OS (23.8 months), comparable with the overall mCRC population [Salvatore and Loupakis, 2012]. However, the significant and independent worse prognostic value of KRAS (58 out of 202 patients) and BRAF mutation (6 out of 292 patients) was recently demonstrated in patients with resection of CLM (hazard ratio [HR] 2.13, 95\% confidence interval [CI] 1.25-3.65 for KRAS mut and HR 6.245, $p<0.003$ for BRAF mut) [Karagkounis and Torbenson, 2012; Teng et al. 2012].

\section{Determination of resectability}

Criteria for resectability are not well defined. Several approaches have been published but with as yet no consensus [Nordlinger et al. 2009; Poston et al. 2008; Yamaguchi et al. 2008]. Concordance in determination of resectability could be achieved in only $64.5 \%$ of cases in the CELIM trial with a board of surgeons blinded for point-in-time of imaging (baseline or after treatment), patients' clinical outcome and the vote of the other participants, whereas critical disagreement (resectable versus nonresectable) was reported in $6.8 \%$ [Folprecht et al. 2010]. In the EORTC 40983 trial, patients with up to four potentially resectable liver metastases and no evidence of extrahepatic disease were included [Nordlinger et al. 2008].

Recent clinical trials include patients depending on resectability determined by a surgeon with hepatic surgery expertise [ClinicalTrials.gov identifier: e.g. NCT01540435, NCT01266187]. In general, limitations for liver surgery are remnant liver of less than $30 \%$, unfavourable location, comorbidity excluding major surgery, aggressive tumour biology and/or the presence of extrahepatic disease. Although number (more than four) and size $(>5 \mathrm{~cm})$ have an impact on prognosis [Yamaguchi et al. 2008], patients could still be deemed resectable and achieve long-term survival with these characteristics. Furthermore, positive margins (R1 resection) seem to be less important for outcome after response to preoperative chemotherapy [Tanaka et al. 2011]. Localized (hepatoduodenal ligament, retropancreatic area) rather than distant (celiac, para-aortic) lymph node metastases does not preclude from long-term 
Table 1. Results from EORTC 40983 trial [Nordlinger et al. 2008].

\begin{tabular}{llllll} 
Patients & $\begin{array}{l}\text { Perioperative } \\
\text { CT }\end{array}$ & $\begin{array}{l}\text { Surgery } \\
\text { alone }\end{array}$ & $\begin{array}{l}\text { Difference for } 3 \text { years } \\
\text { PFSR }\end{array}$ & HR & $\begin{array}{l}\text { Difference for } 5 \text { years } \\
\text { OSR }\end{array}$ \\
\hline All & 182 & 182 & $+7.3 \%(28.1 \%$ to $35.4 \%)$ & $0.79(0.62-1.02)$ & $+3.4 \%(51.2 \%$ to $47.8 \%)$ \\
Eligible & 171 & 171 & $+8.1 \%(28.1 \%$ to $36.2 \%)$ & $0.77(0.60-1.00)$ & $+4.1 \%(52.4 \%$ to $48.3 \%)$ \\
Resected & 151 & 152 & $+9.2 \%(33.2 \%$ to $42.4 \%)$ & $0.73(0.55-0.97)$ & \\
\hline
\end{tabular}

survival (5-yOSR: 25-30\%) after resection of CLM and lymph nodes [Adam et al. 2008; de Haas et al. 2008; Pulitano et al. 2012]. With regard to evolving surgical techniques (e.g. in situ split enabling potential complete and macroscopically curative resections of CLM even in case of disseminated and bilobar disease), technical resectability might often be given, although not every resection might be biologically meaningful.

\section{Systemic treatment of upfront clearly RO resectable CLM}

The role of perioperative treatment in resectable CLM, especially the point in time (preoperative versus postoperative), mode (systemic versus local) and intensity (monotherapy versus combination) is still a matter of debate. Although postoperative therapy after resection of CLM has become an accepted standard of care in many parts of the world, data on the benefit are limited. There are only a few randomized trials comparing preoperative and/or postoperative intravenous chemotherapy or hepatic artery infusion with or without intravenous chemotherapy to surgery alone. Moreover, in adjuvant systemic chemotherapy none of the conducted clinical trials achieved the recruitment goal, leading to solely premature data.

Current guidelines such as European Society for Medical Oncology (ESMO) consensus guidelines recommend upfront surgery for a single liver metastasis with a diameter of up to $2 \mathrm{~cm}$ and good prognosis, whereas for larger single or multiple, clearly R0 resectable CLM preoperative chemotherapy should be administered [Schmoll et al. 2012].

\section{Perioperative systemic treatment}

The EORTC 40983 trial accrued 364 patients to be randomized between surgery alone and chemotherapy with 5-fluorouracil (5FU) and oxaliplatin (FOLFOX4 regimen), administered 3 months preoperatively and postoperatively [Nordlinger et al. 2008]. Patients had to be technically resectable, as stated by computed tomography (CT) scan, with a maximum of 4 CLM and no prior treatment with chemotherapy. Patients' characteristics were favourable, with more than $50 \%$ of patients bearing only one CLM and about $80 \%$ with up to two, respectively. A significant increase in PFS rate at 3 years from $33.2 \%$ to $42.4 \%$ was shown in the group of patients $(n=171)$ receiving chemotherapy and resection of CLM (HR 0.73, $95 \%$ CI $0.55-0.97)$. In the randomized population ('intent-to-treat analyses'), the difference was not statistically significant due to ineligibility of $6 \%$ of patients (HR $0.79,95 \%$ CI $0.62-1.02$ ), but still favouring the chemotherapy arm (Table 1). Recent updated OS analyses (secondary endpoint) revealed a small albeit nonsignificant benefit in 5 -yOSR of $4.1 \%$ in all eligible patients, corresponding to a median OS prolongation of 9 months (55 versus 64 months) [Nordlinger and Sorbye, 2012]. However, the trial was not powered to detect an OS benefit.

No unusual toxicities occurred in the chemotherapy arm. Nearly $80 \%$ of patients completed the preoperative part. After resection, $76 \%$ of patients were receiving postoperative treatment with $52 \%$ of patients $(n=80)$ completing the six scheduled postoperative cycles.

During preoperative chemotherapy main grade $3 / 4$ toxicities were nausea and vomiting $4 \%$, diarrhoea $8 \%$, stomatitis $7 \%$, sensory neuropathy $2 \%$ and neutropenia $18 \%$ (febrile $2 \%$ ). Postoperative reversible complications were significantly higher (25 versus $16 \%, p=0.04$ ) in the chemotherapy group. Particularly intra-abdominal infections, biliary fistulas with an output of more than 100 $\mathrm{ml}$ per day for more than 10 days and hepatic failure with a bilirubin-elevation grade $3 / 4$ for more than 3 days occurred more often in the perioperative group. There was no impact on postoperative death rate ( $1 \%$ in both arms). During postoperative chemotherapy, toxicities were similar with a 
Table 2. Results for adjuvant treatment after resection of stage IV disease.

\begin{tabular}{|c|c|c|c|c|c|c|}
\hline Trial & $\begin{array}{l}\text { Number of } \\
\text { patients }\end{array}$ & Ro & Postoperative treatment & $\begin{array}{l}\text { Absolute difference for } 5 \text { years } \\
\text { OSR or median OS }\end{array}$ & $\operatorname{HR}(95 \% \mathrm{CI})$ & $p$ value \\
\hline Langer et al. [2002] & 129 & $\mathrm{nr}$ & $5 F U / F A$ & +10 months (43 versus 53 ) & $1.30(0.71-2.36)$ & 0.39 \\
\hline Portier et al. [2006] & 171 & 171 & $5 F U / F A$ & +16 months ( 46.4 versus 62.1 ) & $0.73(0.48-1.10)$ & 0.13 \\
\hline Ychou et al. [2009] & 321 & 321 & LV5FU versus FOLFIRI & $\begin{array}{l}+1 \%(71.6 \% \text { versus } 72.7 \% \text { ) } \\
\text { (3 years OSR) }\end{array}$ & $1.09(0.72-1.64)$ & 0.69 \\
\hline Voest et al. [2011] & 79 & 79 & $\begin{array}{l}\text { CAPOX ( } 6 \text { months) versus } \\
\text { CAPOX ( } 6 \text { months) + beva } \\
\text { (12 months) }\end{array}$ & $\begin{array}{l}+18 \% \text { ( } 52 \% \text { versus } 70 \% \text { ) } \\
\text { (2-year DFS rate) }\end{array}$ & $\mathrm{nr}$ & 0.074 \\
\hline
\end{tabular}

higher rate of grade 3 sensory neuropathy (10\%) and other neurological toxicities $(12 \%)$ due to the higher cumulative dose of oxaliplatin.

The results of the EORTC 40983 trial consequently led to the EORTC 40051 'BOS' study with FOLFOX and cetuximab \pm bevacizumab, which was stopped after disadvantageous results for the combination of epidermal growth factor receptor (EGFR) and vascular endothelial growth factor (VEGF) antibodies in CAIRO2 and PACCE [Hecht et al. 2009; Tol et al. 2009b].

\section{Postoperative systemic treatment}

Already the standard of care in patients with stage III disease (and some stage II), data for adjuvant treatment after resection of stage IV disease are still limited (Table 2). Two randomized phase III trials have compared adjuvant systemic chemotherapy with 5FU/folinic acid (FA) after resection of CLM to surgery alone, but both closed prematurely due to slow accrual. A small but significant improvement in recurrence free survival rate (RFSR) could be shown in the FFCD trial at the time of closure, with a 5-year RFSR of 26.7 versus $33.5 \%$ ( $p=$ 0.028), favouring the group with adjuvant treatment [Portier et al. 2006]. Although the 5-yOSR increased from $41.1 \%$ to $51.1 \%$, this difference was not significant $(p=0.13)$. The ENG (EORTC/NCICTG/GIVIO) trial, whose results are still not fully published, displayed a nonsignificant trend towards a prolonged RFS with a median of 39 versus 20 months $(p=0.35)$ and an increased OS with a median of 53 versus 43 months $(p=0.39)$ [Langer et al. 2002]. A combined analysis of both trials $(n=$ 278) showed a nonsignificant prolongation of median disease-free survival (DFS) from 18.8 to
27.9 months $(p=0.058)$ and OS from 47.3 to 62.2 months $(p=0.095)$ [Mitry et al. 2008]. However, both trials were using a noncontemporary $5 \mathrm{FU}$ bolus regimen, which is more toxic and possibly not active enough to reveal a significant increase in this limited number of patients. Postoperative treatment with $5 \mathrm{FU}$ plus irinotecan in a prematurely stopped, randomized phase III trial revealed no additional benefit over $5 \mathrm{FU}$ alone in terms of OS (3-year survival rate of 73 versus $72 \%$ ) [Ychou et al. 2009a]. Regarding median DFS, there was a nonsignificant increase of 3 months $(21.6$ versus 24.7 months, HR 0.90 , 95\% CI 0.67-1.2, $p=$ $0.47)$. However, with overall 306 treated patients the trial might have been insufficiently powered to demonstrate a significant DFS benefit. Moreover, the lack of a clear benefit might also be related to the relatively poor efficacy, as shown in the adjuvant treatment of stage III colorectal disease with $5 \mathrm{FU} /$ FA plus irinotecan (FOLFIRI) [Saltz et al. 2007; Van Cutsem et al. 2009; Ychou et al. 2009b]. Recently results of the again prematurely closed Dutch HEPATICA trial were presented, demonstrating a DFS benefit after 2 years of $18 \%(70 \%$ versus $52 \%$ ) in favour of 12 months bevacizumab with 6 months chemotherapy (capecitabine and oxaliplatin) compared with 6 months chemotherapy alone after R0 resection of CLM in 79 patients [Voest and Snoeren, 2011]. Further follow up might reveal whether this benefit is only transient, as demonstrated for bevacizumab containing adjuvant treatment after resection of stage II or III colon cancer [Allegra et al. 2011; de Gramont, Van Cutsem, et al., 2012]. It is questionable, however, whether further results regarding adjuvant chemotherapy will be available as further postoperative trials (ADHOC, NSABP C-09 and HEPATICA) were closed due to insufficient accrual. 
Table 3. Results following induction treatment in not RO, but potentially resectable, CLM after chemotherapy-induced downsizing.

\begin{tabular}{|c|c|c|c|c|c|c|}
\hline Trial & Treatment & $\begin{array}{l}\text { No. of } \\
\text { patients }\end{array}$ & $\mathrm{RR}(\%)$ & RR ('liver only') & $\mathrm{RO}$ rate all & $\begin{array}{l}\mathrm{RO} \text { rate } \\
\text { 'liver only' }\end{array}$ \\
\hline Van Cutsem et al. [2011] & FOLFIRI+cet & $316^{*}$ & $57.3^{*}$ & $70.6 *(n=68)$ & 5.1 & 13.2 \\
\hline Bokemeyer et al. [2011] & FOLFOX+cet & $82 *$ & $57^{*}$ & $76 *(n=25)$ & 7.3 & 16 \\
\hline Maughan et al. [2011] & 5FU/cape+oxali+cet & $362^{*}$ & $64^{*}$ & $\mathrm{nr}$ & $\mathrm{nr}$ & $\mathrm{nr}$ \\
\hline Douillard et al. [2011] & FOLFOX+pan & 593 & $57 *$ & $\mathrm{nr}$ & $\mathrm{nr}$ & 28 \\
\hline Hurwitz et al. [2004] & IFL+beva & 402 & 45 & $\mathrm{nr}$ & $<2 \%$ & $\mathrm{nr}$ \\
\hline Saltz et al. [2007, 2008] & 5FU/cape+oxali+beva & 699 & 47 & $\mathrm{nr}$ & 6.3 & 12.3 \\
\hline Falcone et al. [2007] & FOLFOXIRI & 122 & 66 & $\mathrm{nr}$ & 15 & 36 \\
\hline
\end{tabular}

\section{Ongoing trials in $\mathrm{RO}$ resectable CLM}

Several randomized trials are currently ongoing evaluating intensified perioperative regimens, for example, CRUK06/031 (FOLFOX \pm cetuximab) and EORTC BOS-2 (FOLFOX alone versus FOLFOX plus EGFR antibody or FOLFOX plus bevacizumab), both in KRAS wild-type patients, as well as intensified perioperative versus postoperative approaches such as the German PANTER (FOLFOX plus cetuximab 3 months preoperative and postoperative versus 6 months postoperative) in KRAS wild-type, the Australian ATTACHE (FOLFOX/XELOX/FOLFIRI plus bevacizumab 3 months preoperative and postoperative versus 6 months postoperative) or PERIMAX (FOLFOXIRI plus bevacizumab 3 months preoperative and postoperative versus FOLFOX 6 months postoperative).

\section{Induction treatment in not RO, but potentially resectable, CLM after chemotherapy-induced downsizing}

The group of patients with potentially resectable metastases warrants a highly active, neoadjuvant or induction chemotherapy that aims to achieve resectability, with tumour shrinkage as the most important goal. Regarding the reported Response Evaluation Criteria in Solid Tumors (RECIST) overall response rates (ORR) displayed in clinical trials in the first-line treatment for $\mathrm{mCRC}$, a threedrug combination containing EGFR-targeting antibody in KRAS wild-type patients or bevacizumab or a three-drug chemotherapy regimen (FOLFOXIRI) should be chosen [Bokemeyer et al. 2011; Douillard and Siena, 2011; Falcone et al. 2007; Hurwitz et al. 2004; Maughan et al. 2011; Saltz et al. 2008; van Cutsem et al. 2011] (Table 3). These trials included all patients with
mCRC, and the benefit for "liver only" disease cannot be assessed properly.

Recent data from single arm phase II trials with different $5 \mathrm{FU} / \mathrm{FA}$, irinotecan and oxaliplatin schedules in combination with either bevacizumab or EGFR antibodies consistently reported a high ORR of about $80 \%$ [Assenat et al. 2011; Bruera et al. 2010; Garufi et al. 2010; Lonardi and Fornaro, 2012; Masi et al. 2010]. In particular, the four-drug regimens with EGFR antibodies were compromised by grade 3/4 diarrhoea, leading to dose reduction of chemotherapy with maintained efficacy. In one trial with FOLFOXIRI plus bevacizumab, the ORR of $80 \%$ in 'liver only' patients resulted in a favourable $\mathrm{R} 0$ resection rate of $26 \%(n=15)$ [Masi et al. 2010].

Several trials including mCRC patients without extrahepatic disease have shown ORR in the range of 71 to $78 \%$ with current three-drug regimens (5FU, irinotecan, and oxaliplatin (FOLFIRINOX), capecitabine plus oxaliplatin (CAPOX) plus bevacizumab, FOLFOX/FOLFIRI plus cetuximab [Folprecht and Gruenberger, 2010; Gruenberger et al. 2008; Wong et al. 2011; Ychou et al. 2008]. Although the above-mentioned limitations about determination of resectability need to be taken into account, the CELIM and BOXER trial reported rates of secondary resectability of 28 and $40 \%$, respectively [Folprecht et al. 2010; Wong et al. 2011]. Recently, survival analysis of the CELIM trial demonstrated a significant PFS benefit of 15.4 versus 8.9 months (HR 2.07, $p=0.001$ ) and OS improvement of 46.7 versus 27.3 months (HR 2.34, $p=0.002$ ) in favour of R0 resected patients $(n=36)$ compared with those with no or $\mathrm{R} 1 / 2$ resection $(n=70)$; this further strengthens the approach of aiming at and performing 
secondary resection if feasible [Folprecht and Gruenberger, 2012].

If resectability cannot be achieved by systemic chemotherapy alone, combination with hepatic artery infusion might be a possible approach as recently demonstrated in a small group of 61 treated patients with a resection rate of $44 \%$ [Kemeny and Fong, 2012].

Ongoing trials are evaluating different 5FU/FA, irinotecan and oxaliplatin regimens in patients with liver-limited disease with either panitumumab or cetuximab in KRAS wild-type or bevacizumab (e.g. Austrian MO18725, CELIM 2, PRODIGE 14, VOLFI).

\section{Controversies about neoadjuvant/ induction treatment}

Although tumour shrinkage is important in order to achieve technical resectability and RECIST response rates are likely to correlate well with the percentage of resected or 'converted' patients, patient prognosis may be determined even more by the effect of treatment on tumor tissue and -biology than by the achievement of resectability alone. Accordingly, good correlations of pathohistological response to preoperative chemotherapy, which seemed to be increased with bevacizumab-containing combinations, were reported with OS from different cohorts of patients after resection of CLM [Blazer et al. 2008; Klinger et al. 2010; Rubbia-Brandt et al. 2007]. These have to be kept in mind in case of cross-trial comparison for response rates.

One of the major drawbacks in neoadjuvant treatment is the change into irresectability in cases of progression during first-line induction chemotherapy. In the EORTC 40983 trial, 12 (7\%) patients experienced progressive disease during preoperative chemotherapy leading to irresectability in 8 patients, half of whom presented with new lesions [Nordlinger et al. 2008]. However, the switch to irresectability reveals aggressive tumour biology and predicts a worse outcome even with resection, or defines inadequate chemotherapy with a 2 drug regimen only, or both.

Another issue is the complete radiological response during neoadjuvant treatment, as complete clinical response does not mean complete pathological response [Benoist et al. 2006; Gaujoux et al. 2011]. Therefore patients should be closely monitored during preoperative chemotherapy and ideally resected before complete remission. If an anatomical resection can be performed, complete response is not a major problem because resection will be based on initial sites of liver metastases. In cases of complete response on CT and no option for anatomical resection, different imaging methods might be used [magnetic resonance imaging (MRI), positron emission tomography (PET) scan, contrast-enhanced ultrasound] or resection might be delayed until relapse occurs.

Chemotherapy can result in severe damage of liver tissue. Whereas oxaliplatin-based combinations are associated with increased risk of vascular lesions, irinotecan-containing regimens have been associated with increased risk of steatohepatitis, especially in obese patients with a body mass index (BMI) over $25 \mathrm{~kg} / \mathrm{m}^{2}$ [Aloia et al. 2006; Benoist and Nordlinger, 2009; Vauthey et al. 2006]. The clinical impact of chemotherapy-associated histological changes seems to favour oxaliplatin in the neoadjuvant setting. Increased risk of perioperative bleeding, postoperative complication rate and poorer functional reserve was found to be associated with vascular lesions. Although influence on morbidity is still controversial, mortality was not affected [Nakano et al. 2008; Vauthey et al. 2006]. In the EORTC 40983 trial, the perioperative treatment resulted in a similar postoperative death rate to surgery alone, with a significantly higher rate of reversible postoperative complications $(p=0.04)$. Higher risk of complications and an increased 90-day mortality [1.6\% versus $14.7 \%$, odds ratio (OR) 10.5] was reported for irinotecan-associated steatohepatitis [Vauthey et al. 2006]. The addition of bevacizumab to neoadjuvant oxaliplatin-based treatment is feasible, does not limit the ability of liver regeneration, and even seems to protect the liver from oxaliplatininduced liver toxicity [Gruenberger et al. 2008; Ribero et al. 2007].

Duration of preoperative treatment seem to have a major impact on perioperative morbidity and prognosis. Several retrospective analyses suggested an increased postoperative morbidity (19\% versus $54 \% p=0.047$ for $</ \geq 6$ cycles, higher rate of liver insufficiency ( $4 \%$ versus $11 \%, p=0.035$ ) for $<>\geq 9$ cycles and higher rate of reoperation $(0 \%$ versus $11 \%, p=0.02$ ) and longer hospitalization ( 11 versus 15 days, $p=0.02$ ) for $\leq />12$ cycles [Aloia et al. 2006; Karoui et al. 2006; Kishi et al. 2010]. Moreover, the recent liver metastases survey showed a DFS detriment for patients with more than six cycles of preoperative chemotherapy 
[Adam and Barroso, 2011]. Duration of preoperative chemotherapy should therefore be limited to 3 months in case of initially $\mathrm{R} 0$ resectable disease (followed by 3 months postoperative treatment) or as short as possible in case of initially unresectable disease. Although liver regeneration and postoperative complication rates do not seem to be affected by preoperative bevacizumab treatment, the interval to surgery is a critical factor with regard to wound-healing complications and bleeding [Erinjeri et al. 2011; Kesmodel et al. 2008; Wicherts et al. 2011]. According to the summary of product characteristics (SmPC), the minimum interval between bevacizumab administration and operation should be 4 weeks; thus the last preoperative chemotherapy might be applied without bevacizumab.

Besides treatment-naïve patients with synchronous liver metastases, a large group of patients with metachronous disease will have received adjuvant chemotherapy. Whereas adjuvant single-agent fluoropyrimidines do not influence choice of treatment, the interval between oxaliplatin-based adjuvant chemotherapy and hepatic recurrence is important for selection of neoadjuvant or induction chemotherapy. In cases of recurrence within 6 months after completion of adjuvant oxaliplatin, irinotecan-based chemotherapy might be more appropriate even in clearly R0 resectable liver metastases.

\section{Conclusion}

The prognosis of patients with CLM depends on their access to an optimal multimodal treatment. Systemic perioperative chemotherapy administered preoperatively and/or postoperatively has been shown to reduce recurrence and prolong survival in initially clearly $\mathrm{R} 0$ resectable disease. Besides the subgroup of patients with a single small CLM, for whom upfront resection should be performed, perioperative chemotherapy for 3 months preoperatively and 3 months postoperatively should be administered.

For not R0 but potentially resectable CLM after chemotherapy, an aggressive treatment approach is broadly accepted with at least chemotherapy triplets or chemotherapy doublets in combination with EGFR or VEGF antibodies. This approach might possibly also be useful for patients with R0 resectable CLM, as DFS is still poor after curative resection. Current trials reflect ongoing discussions about the optimal treatment approach for CLM comparing either perioperative versus postoperative, or two versus three or even four drug regimens in resectable and three versus four drug regimens in potentially resectable liver-limited disease.

\section{Funding}

This research received no specific grant from any funding agency in the public, commercial, or notfor-profit sectors.

\section{Conflict of interest statement}

AS has received honoraria from Roche and Merck Serono. HJS has performed a consultant or advisory role at Roche, received an honorarium from Roche, and research support from Roche and Merck Serono.

\section{References}

Adam, R. and Barroso, E. (2011) Impact of the type and modalities of preoperative chemotherapy on the outcome of liver resection for colorectal metastases. f Clin Oncol 29(Suppl.): abstract 3519.

Adam, R., de Haas, R., Wicherts, D., Aloia, T., Delvart, V., Azoulay, D. et al. (2008) Is hepatic resection justified after chemotherapy in patients with colorectal liver metastases and lymph node involvement? f Clin Oncol 26: 3672-3680.

Allegra, C., Yothers, G., O’Connell, M., Sharif, S., Petrelli, N., Colangelo, L. et al. (2011) Phase III trial assessing bevacizumab in stages II and III carcinoma of the colon: results of NSABP protocol C-08. $\mathcal{F}$ Clin Oncol 29: 11-16.

Aloia, T., Sebagh, M., Plasse, M., Karam, V., Levi, F., Giacchetti, S. et al. (2006) Liver histology and surgical outcomes after preoperative chemotherapy with fluorouracil plus oxaliplatin in colorectal cancer liver metastases. f Clin Oncol 24: 4983-4990.

Assenat, E., Desseigne, F., Thezenas, S., Viret, F., Mineur, L., Kramar, A. et al. (2011) Cetuximab plus FOLFIRINOX (ERBIRINOX) as first-line treatment for unresectable metastatic colorectal cancer: a phase II trial. Oncologist 16: 1557-1564.

Benoist, S., Brouquet, A., Penna, C., Julie, C., El Hajjam, M., Chagnon, S. et al. (2006) Complete response of colorectal liver metastases after chemotherapy: does it mean cure? F Clin Oncol 24: 3939-3945.

Benoist, S. and Nordlinger, B. (2009) The role of preoperative chemotherapy in patients with resectable colorectal liver metastases. Ann Surg Oncol 16: 2385-2390.

Blazer, D. III, Kishi, Y., Maru, D., Kopetz, S., Chun, Y., Overman, M. et al. (2008) Pathologic 
response to preoperative chemotherapy: a new outcome end point after resection of hepatic colorectal metastases. F Clin Oncol 26: 5344-5351.

Bokemeyer, C., Bondarenko, I., Hartmann, J., de Braud, F., Schuch, G., Zubel, A. et al. (2011) Efficacy according to biomarker status of cetuximab plus FOLFOX-4 as first-line treatment for metastatic colorectal cancer: the OPUS study. Ann Oncol 22: 1535-1546.

Bokemeyer, C., Cutsem, E., Rougier, P., Ciardiello, F., Heeger, S., Schlichting, M. et al. (2012) Addition of cetuximab to chemotherapy as first-line treatment for KRAS wild-type metastatic colorectal cancer: pooled analysis of the CRYSTAL and OPUS randomised clinical trials. Eur f Cancer 48: 1466-1475.

Bruera, G., Santomaggio, A., Cannita, K., Baldi, P., Tudini, M., De Galitiis, F. et al. (2010) 'Poker' association of weekly alternating 5-fluorouracil, irinotecan, bevacizumab and oxaliplatin (FIr-B/FOx) in first line treatment of metastatic colorectal cancer: a phase II study. BMC Cancer 10: 567.

de Gramont, A., Van Cutsem, E. et al. (2012) Bevacizumab plus oxaliplatin-based chemotherapy as adjuvant treatment for colon cancer (AVANT): a phase 3 randomised controlled trial. Lancet Oncol 13(12): 1225-1233.

De Haas, R., Wicherts, D., Flores, E., Azoulay, D., Castaing, D. and Adam, R. (2008) R1 resection by necessity for colorectal liver metastases: is it still a contraindication to surgery? Ann Surg 248: 626-637.

De Jong, M., Pulitano, C., Ribero, D., Strub, J., Mentha, G., Schulick, R. et al. (2009) Rates and patterns of recurrence following curative intent surgery for colorectal liver metastasis: an international multi-institutional analysis of 1669 patients. Ann Surg 250: 440-448.

Douillard, J. and Siena, S. (2011) Final results from PRIME: randomized phase III study of panitumumab (pmab) with FOLFOX4 for first-line metastatic colorectal cancer (mCRC). F Clin Oncol 29(Suppl.): abstract 3510.

Erinjeri, J., Fong, A., Kemeny, N., Brown, K., Getrajdman, G. and Solomon, S. (2011) Timing of administration of bevacizumab chemotherapy affects wound healing after chest wall port placement. Cancer 117: 1296-1301.

Falcone, A., Ricci, S., Brunetti, I., Pfanner, E., Allegrini, G., Barbara, C. et al. (2007) Phase III trial of infusional fluorouracil, leucovorin, oxaliplatin, and irinotecan (FOLFOXIRI) compared with infusional fluorouracil, leucovorin, and irinotecan (FOLFIRI) as first-line treatment for metastatic colorectal cancer: the Gruppo Oncologico Nord Ovest. f Clin Oncol 25: 1670-1676.

Ferlay, J., Shin, H., Bray, F., Forman, D., Mathers, C. and Parkin, D. (2010) Estimates of worldwide burden of cancer in 2008: GLOBOCAN 2008. Int $\mathcal{F}$ Cancer 127: 2893-2917.

Folprecht, G. and Gruenberger, T. (2012) Survival with cetuximab/FOLFOX or cetuximab/FOLFIRI of patients with nonresectable colorectal liver metastases in the CELIM study. F Clin Oncol 30(Suppl. 4): abstract 540.

Folprecht, G., Gruenberger, T., Bechstein, W., Raab, H., Lordick, F., Hartmann, J. et al. (2010) Tumour response and secondary resectability of colorectal liver metastases following neoadjuvant chemotherapy with cetuximab: the CELIM randomised phase 2 trial. Lancet Oncol 11: 38-47.

Fong, Y., Fortner, J., Sun, R., Brennan, M. and Blumgart, L. (1999) Clinical score for predicting recurrence after hepatic resection for metastatic colorectal cancer: analysis of 1001 consecutive cases. Ann Surg 230: 309-318; discussion: 318-321.

Garufi, C., Torsello, A., Tumolo, S., Ettorre, G., Zeuli, M., Campanella, C. et al. (2010) Cetuximab plus chronomodulated irinotecan, 5-fluorouracil, leucovorin and oxaliplatin as neoadjuvant chemotherapy in colorectal liver metastases: POCHER trial. Br F Cancer 103: 1542-1547.

Gaujoux, S., Goere, D., Dumont, F., Souadka, A., Dromain, C., Ducreux, M. et al. (2011) Complete radiological response of colorectal liver metastases after chemotherapy: what can we expect? Dig Surg 28: 114-120.

Gruenberger, B., Tamandl, D., Schueller, J., Scheithauer, W., Zielinski, C., Herbst, F. et al. (2008) Bevacizumab, capecitabine, and oxaliplatin as neoadjuvant therapy for patients with potentially curable metastatic colorectal cancer. F Clin Oncol 26: 1830-1835.

Hecht, J., Mitchell, E., Chidiac, T., Scroggin, C., Hagenstad, C., Spigel, D. et al. (2009) A randomized phase IIIB trial of chemotherapy, bevacizumab, and panitumumab compared with chemotherapy and bevacizumab alone for metastatic colorectal cancer. f Clin Oncol 27: 672-680.

Hughes, K., Simon, R., Songhorabodi, S., Adson, M., Ilstrup, D., Fortner, J. et al. (1986) Resection of the liver for colorectal carcinoma metastases: a multiinstitutional study of patterns of recurrence. Surgery 100: 278-284.

Hurwitz, H., Fehrenbacher, L., Novotny, W., Cartwright, T., Hainsworth, J., Heim, W. et al. (2004) Bevacizumab plus irinotecan, fluorouracil, and leucovorin for metastatic colorectal cancer. $N$ Engl $\mathcal{F}$ Med 350: 2335-2342.

Jemal, A., Bray, F., Center, M., Ferlay, J., Ward, E. and Forman, D. (2011) Global cancer statistics. $C A$ Cancer f Clin 61: 69-90. 
Karagkounis, G. and Torbenson, M. (2012) Incidence and prognostic impact of KRAS and BRAF mutations in patients undergoing liver surgery for colorectal metastases. F Clin Oncol 30: abstract 3616.

Karoui, M., Penna, C., Amin-Hashem, M., Mitry, E., Benoist, S., Franc, B. et al. (2006) Influence of preoperative chemotherapy on the risk of major hepatectomy for colorectal liver metastases. Ann Surg 243: 1-7.

Kemeny, N. and Fong, Y. (2012) Is conversion to resection possible with hepatic arterial infusion (HAI) and systemic (SYS) even in previously treated patients (pts) with unresectable colorectal liver metastases (UnCLM)? f Clin Oncol 30: abstract 3577.

Kesmodel, S., Ellis, L., Lin, E., Chang, G., Abdalla, E., Kopetz, S. et al. (2008) Preoperative bevacizumab does not significantly increase postoperative complication rates in patients undergoing hepatic surgery for colorectal cancer liver metastases. $\mathcal{F}$ Clin Oncol 26: 5254-5260.

Kishi, Y., Zorzi, D., Contreras, C., Maru, D., Kopetz, S., Ribero, D. et al. (2010) Extended preoperative chemotherapy does not improve pathologic response and increases postoperative liver insufficiency after hepatic resection for colorectal liver metastases. Ann Surg Oncol 17: 2870-2876.

Klinger, M., Tamandl, D., Eipeldauer, S., Hacker, S., Herberger, B., Kaczirek, K. et al. (2010) Bevacizumab improves pathological response of colorectal cancer liver metastases treated with XELOX/FOLFOX. Ann Surg Oncol 17: 2059-2065.

Kopetz, S., Chang, G., Overman, M., Eng, C., Sargent, D., Larson, D. et al. (2009) Improved survival in metastatic colorectal cancer is associated with adoption of hepatic resection and improved chemotherapy. F Clin Oncol 27: 3677-3683.

Langer, B., Bleiberg, H., Labianca, R., Shepherd, L., Nitti, D., Marsoni, S. et al. (2002) Fluorouracil (FU) plus 1-leucovorin (1-LV) versus observation after potentially curative resection of liver or lung metastases from colorectal cancer (CRC): results of the ENG (EORTC/NCIC CTG/GIVIO) randomized trial. Proc Am Soc Clin Oncol 21: 2002 (abstract 592).

Lonardi, S. and Fornaro, L. (2012) Phase II study of panitumumab $(\mathrm{P})$ in combination with FOLFOXIRI as first-line treatment of metastatic colorectal cancer (mCRC): activity in molecularly selected patients (pts). F Clin Oncol 30: abstract 3555.

Mantke, R., Schmidt, U., Wolff, S., Kube, R. and Lippert, H. (2012) Incidence of synchronous liver metastases in patients with colorectal cancer in relationship to clinico-pathologic characteristics. Results of a German prospective multicentre observational study. Eur F Surg Oncol 38: 259-265.
Masi, G., Loupakis, F., Salvatore, L., Fornaro, L., Cremolini, C., Cupini, S. et al. (2010) Bevacizumab with FOLFOXIRI (irinotecan, oxaliplatin, fluorouracil, and folinate) as first-line treatment for metastatic colorectal cancer: a phase 2 trial. Lancet Oncol 11: 845-852.

Maughan, T., Adams, R., Smith, C., Meade, A., Seymour, M., Wilson, R. et al. (2011) Addition of cetuximab to oxaliplatin-based first-line combination chemotherapy for treatment of advanced colorectal cancer: results of the randomised phase $3 \mathrm{MRC}$ COIN trial. Lancet 377: 2103-2114.

Mitry, E., Fields, A., Bleiberg, H., Labianca, R., Portier, G., Tu, D. et al. (2008) Adjuvant chemotherapy after potentially curative resection of metastases from colorectal cancer: a pooled analysis of two randomized trials. $\mathcal{F}$ Clin Oncol 26: 4906-4911.

Morris, E., Forman, D., Thomas, J., Quirke, P., Taylor, E., Fairley, L. et al. (2010) Surgical management and outcomes of colorectal cancer liver metastases. Br F Surg 97: 1110-1118.

Nakano, H., Oussoultzoglou, E., Rosso, E., Casnedi, S., Chenard-Neu, M.P., Dufour, P. et al. (2008) Sinusoidal injury increases morbidity after major hepatectomy in patients with colorectal liver metastases receiving preoperative chemotherapy. Ann Surg 247: 118-124.

Nordlinger, B. and Sorbye, H. (2012) EORTC liver metastases intergroup randomized phase III study 40983: Long-term survival results. $\mathcal{F}$ Clin Oncol 30 (Suppl.): abstract 3508.

Nordlinger, B., Sorbye, H., Glimelius, B., Poston, G., Schlag, P., Rougier, P. et al. (2008) Perioperative chemotherapy with FOLFOX4 and surgery versus surgery alone for resectable liver metastases from colorectal cancer (EORTC Intergroup trial 40983): a randomised controlled trial. Lancet 371 :

1007-1016.

Nordlinger, B., van Cutsem, E., Gruenberger, T., Glimelius, B., Poston, G., Rougier, P. et al. (2009) Combination of surgery and chemotherapy and the role of targeted agents in the treatment of patients with colorectal liver metastases: recommendations from an expert panel. Ann Oncol 20: 985-992.

Portier, G., Elias, D., Bouche, O., Rougier, P., Bosset, J.F., Saric, J. et al. (2006) Multicenter randomized trial of adjuvant fluorouracil and folinic acid compared with surgery alone after resection of colorectal liver metastases: FFCD ACHBTH AURC 9002 trial. f Clin Oncol 24: 4976-4982.

Poston, G., Figueras, J., Giuliante, F., Nuzzo, G., Sobrero, A., Gigot, J. et al. (2008) Urgent need for a new staging system in advanced colorectal cancer. f Clin Oncol 26: 4828-4833. 
Price, T., Hardingham, J., Lee, C., Weickhardt, A., Townsend, A., Wrin, J. et al. (2011) Impact of KRAS and BRAF gene mutation status on outcomes from the phase III AGITG MAX trial of capecitabine alone or in combination with bevacizumab and mitomycin in advanced colorectal cancer. $\mathcal{F}$ Clin Oncol 29: 2675-2682.

Pulitano, C., Bodingbauer, M., Aldrighetti, L., Choti, M., Castillo, F., Schulick, R. et al. (2012) Colorectal liver metastasis in the setting of lymph node metastasis: defining the benefit of surgical resection. Ann Surg Oncol 19: 435-442.

Ribero, D., Wang, H., Donadon, M., Zorzi, D., Thomas, M., Eng, C. et al. (2007) Bevacizumab improves pathologic response and protects against hepatic injury in patients treated with oxaliplatinbased chemotherapy for colorectal liver metastases. Cancer 110: 2761-2767.

Rubbia-Brandt, L., Giostra, E., Brezault, C., Roth, A., Andres, A., Audard, V. et al. (2007) Importance of histological tumor response assessment in predicting the outcome in patients with colorectal liver metastases treated with neo-adjuvant chemotherapy followed by liver surgery. Ann Oncol 18: 299-304.

Saltz, L., Clarke, S., Diaz-Rubio, E., Scheithauer, W., Figer, A., Wong, R. et al. (2008) Bevacizumab in combination with oxaliplatin-based chemotherapy as first-line therapy in metastatic colorectal cancer: a randomized phase III study. F Clin Oncol 26: 2013-2019.

Saltz, L., Niedzwiecki, D., Hollis, D., Goldberg, R., Hantel, A., Thomas, J. et al. (2007) Irinotecan fluorouracil plus leucovorin is not superior to fluorouracil plus leucovorin alone as adjuvant treatment for stage III colon cancer: results of CALGB 89803. J Clin Oncol 25: 3456-3461.

Salvatore, L. and Loupakis, F. (2012) FOLFOXIRI plus bevacizumab as first-line treatment of BRAF mutant metastatic colorectal cancer patients. $\mathcal{F}$ Clin Oncol 30: abstract 3585.

Schmoll, H. and Sargent, D. (2007) Single agent fluorouracil for first-line treatment of advanced colorectal cancer as standard? Lancet 370: 105-107.

Schmoll, H., van Cutsem, E., Stein, A., Valentini, V., Glimelius, B., Haustermans, K. et al. (2012) ESMO consensus guidelines for management of patients with colon and rectal cancer. A personalized approach to clinical decision making. Ann Oncol 23: 2479-2516.

Tanaka, K., Nojiri, K., Kumamoto, T., Takeda, K. and Endo, I. (2011) R1 resection for aggressive or advanced colorectal liver metastases is justified in combination with effective prehepatectomy chemotherapy. Eur f Surg Oncol 37: 336-343.

Teng, H., Huang, Y., Lin, J., Chen, W., Lin, T., Jiang, J. et al. (2012) BRAF mutation is a prognostic biomarker for colorectal liver metastasectomy. f Surg Oncol 106: 123-129.

Tol, J., Koopman, M., Cats, A., Rodenburg, C., Creemers, G., Schrama, J. et al. (2009b)

Chemotherapy, bevacizumab, and cetuximab in metastatic colorectal cancer. $N$ Engl F Med 360: 563-572.

Tol, J., Nagtegaal, I. and Punt, C. (2009a) BRAF mutation in metastatic colorectal cancer. $N$ Engl $\mathcal{F}$ Med 361: 98-99.

Van Cutsem, E., Dicato, M., Arber, N., Berlin, J., Cervantes, A., Ciardiello, F. et al. (2010) Molecular markers and biological targeted therapies in metastatic colorectal cancer: expert opinion and recommendations derived from the 11th ESMO/ World Congress on Gastrointestinal Cancer, Barcelona, 2009. Ann Oncol 21(Suppl. 6): vi1-vi10.

Van Cutsem, E., Kohne, C., Lang, I., Folprecht, G., Nowacki, M., Cascinu, S. et al. (2011) Cetuximab plus irinotecan, fluorouracil, and leucovorin as first-line treatment for metastatic colorectal cancer: updated analysis of overall survival according to tumor KRAS and BRAF mutation status. F Clin Oncol 29: 2011-2019.

Van Cutsem, E., Labianca, R., Bodoky, G., Barone, C., Aranda, E., Nordlinger, B. et al. (2009) Randomized phase III trial comparing biweekly infusional fluorouracil/leucovorin alone or with irinotecan in the adjuvant treatment of stage III colon cancer: PETACC-3. f Clin Oncol 27: 3117-3125.

Van der Pool, A., Damhuis, R., Ijzermans, J., de Wilt, J., Eggermont, A., Kranse, R. et al. (2012) Trends in incidence, treatment and survival of patients with stage IV colorectal cancer: a populationbased series. Colorectal Dis 14: 56-61.

Vauthey, J., Pawlik, T., Ribero, D., Wu, T., Zorzi, D., Hoff, P. et al. (2006) Chemotherapy regimen predicts steatohepatitis and an increase in 90-day mortality after surgery for hepatic colorectal metastases. $\mathcal{F}$ Clin Oncol 24: 2065-2072.

Voest, E. and Snoeren, N. (2011) A randomized two-arm phase III study to investigate bevacizumab in combination with capecitabine plus oxaliplatin (CAPOX) versus CAPOX alone in post radical resection of patients with liver metastases of colorectal cancer. $\mathcal{F}$ Clin Oncol 29(Suppl.): abstract 3565 .

Wicherts, D., de Haas, R., Sebagh, M., Saenz Corrales, E., Gorden, D., Levi, F. et al. (2011) Impact of bevacizumab on functional recovery and histology of the liver after resection of colorectal metastases. $\mathrm{Br}$ f Surg 98: 399-407.

Wong, R., Cunningham, D., Barbachano, Y., Saffery, C., Valle, J., Hickish, T. et al. (2011) A 
multicentre study of capecitabine, oxaliplatin plus bevacizumab as perioperative treatment of patients with poor-risk colorectal liver-only metastases not selected for upfront resection. Ann Oncol 22: 2042-2048.

Yamaguchi, T., Mori, T., Takahashi, K., Matsumoto, H., Miyamoto, H. and Kato, T. (2008) A new classification system for liver metastases from colorectal cancer in Japanese multicenter analysis. Hepatogastroenterology 55: 173-178.

Ychou, M., Hohenberger, W., Thezenas, S., Navarro, M., Maurel, J., Bokemeyer, C. et al. (2009a) A randomized phase III study comparing adjuvant 5-fluorouracil/folinic acid with FOLFIRI in patients following complete resection of liver metastases from colorectal cancer. Ann Oncol 20: 1964-1970.

Ychou, M., Raoul, J., Douillard, J., GourgouBourgade, S., Bugat, R., Mineur, L. et al. (2009b) A phase III randomised trial of LV5FU2 + irinotecan versus LV5FU2 alone in adjuvant high-risk colon cancer (FNCLCC Accord02/FFCD9802). Ann Oncol 20: 674-680.

Ychou, M., Viret, F., Kramar, A., Desseigne, F., Mitry, E., Guimbaud, R. et al. (2008) Tritherapy with fluorouracil/leucovorin, irinotecan and oxaliplatin (FOLFIRINOX): a phase II study in colorectal cancer patients with non-resectable liver metastases. Cancer Chemother Pharmacol 62: 195-201.
Visit SAGE journals online http://tam.sagepub.com

(SAGE journals 